NIST Technical Note 2031

\title{
Temperature Field Measurements using Thin Filament Pyrometry in a Medium- Scale Methanol Pool Fire
}

\author{
Zhigang Wang \\ Wai Cheong Tam \\ Ki Yong Lee \\ Anthony Hamins
}

This publication is available free of charge from:

https://doi.org/10.6028/NIST.TN.2031 
NIST Technical Note 2031

\title{
Temperature Field Measurements using Thin Filament Pyrometry in a Medium- Scale Methanol Pool Fire
}

\author{
Zhigang Wang* \\ Wai Cheong Tam \\ Ki Yong Lee** \\ Anthony Hamins \\ Fire Research Division \\ Engineering Laboratory \\ permanent addresses: \\ * University of Science and Technology of China, Hefei, China \\ ** Andong National University, Andong, Republic of Korea
}

This publication is available free of charge from:

https://doi.org/10.6028/NIST.TN.2031

November 2018

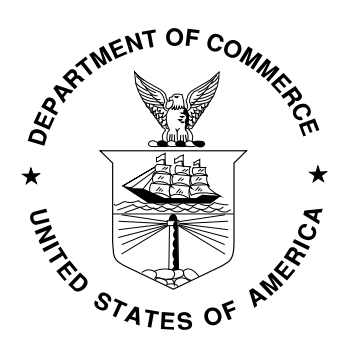

U.S. Department of Commerce Wilbur L. Ross, Jr., Secretary

National Institute of Standards and Technology Walter Copan, NIST Director and Under Secretary of Commerce for Standards and Technology 
Certain commercial entities, equipment, or materials may be identified in this document in order to describe an experimental procedure or concept adequately. Such identification is not intended to imply recommendation or endorsement by the National Institute of Standards and Technology, nor is it intended to imply that the entities, materials, or equipment are necessarily the best available for the purpose.

National Institute of Standards and Technology Technical Note 2031 Natl. Inst. Stand. Technol. Tech. Note 2031, 24 pages (November 2018) CODEN: NTNOEF

This publication is available free of charge from: https://doi.org/10.6028/NIST.TN.2031 


\section{Table of Contents}

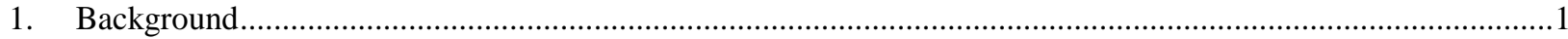

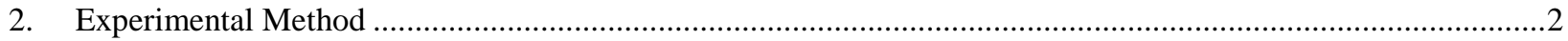

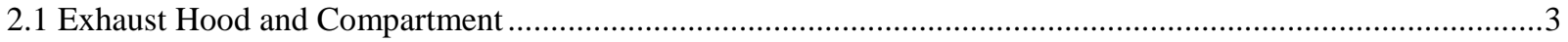

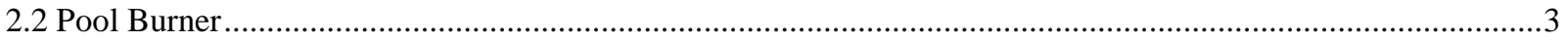

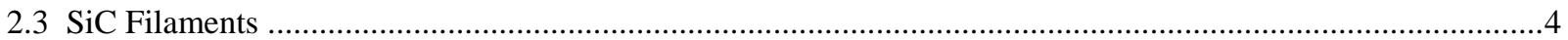

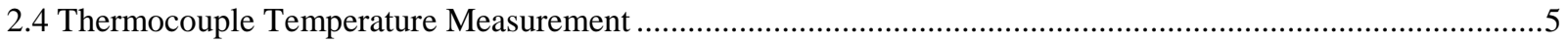

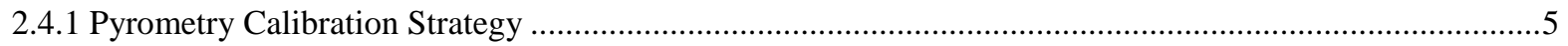

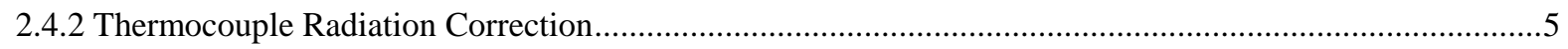

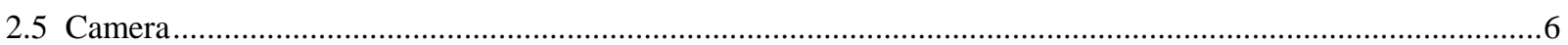

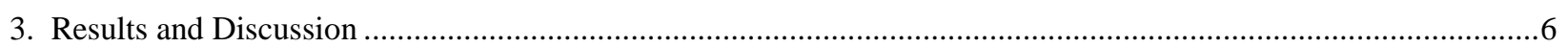

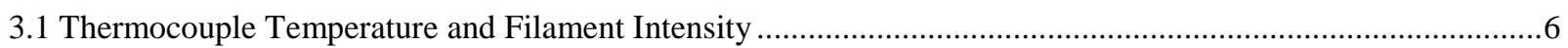

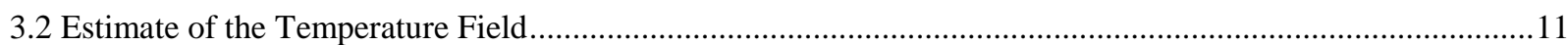

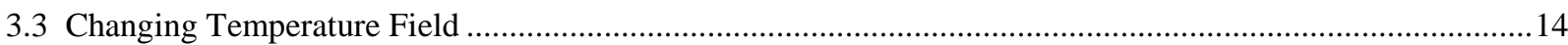

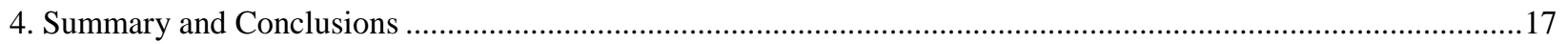

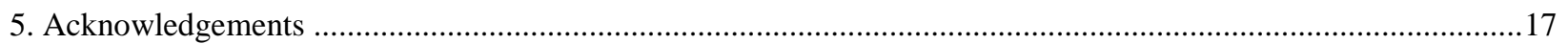

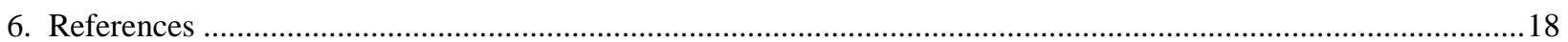




\title{
Temperature Field Measurements using Thin Filament Pyrometry in a Medium-Scale Methanol Pool Fire
}

\begin{abstract}
Measurements were made to characterize the time-varying temperature field in a medium-sized methyl alcohol (methanol; $\mathrm{CH}_{3} \mathrm{OH}$ ) pool fire steadily burning in a quiescent environment.

A digital camera fitted with optical filters and a zoom lens was used to record the high temperature emission intensity of $14 \mu \mathrm{m}$ diameter Type S Silicon-Carbide filaments oriented horizontally at various heights above a central cross-section of a steadily burning $0.30 \mathrm{~m}$ diameter methanol pool fire. The camera optics focused on the filaments with a field of view of approximately $0.3 \mathrm{~m}$ wide by $0.6 \mathrm{~m}$ high. Using the optical camera, the filament was observable only for temperatures larger than $1150 \mathrm{~K}$. Experiments were conducted to collect thousands of frames of $30 \mathrm{~Hz}$ video. In a separate experiment, a $50 \mu \mathrm{m}$ diameter thermocouple was used to acquire 3400 independent temperature measurements at several locations in the high temperature zone of the fire. A correlation was developed between the probability density functions of the radiation-corrected thermocouple measurements and the camera grayscale pixel intensity of the filament for the same fire locations.

Assuming a Gaussian temperature distribution, a fitting routine was used to determine the mean temperature and its variance from the calculated filament temperature. The mean temperatures compared favorably to previously reported measurements. False color maps of temperature were produced characterizing the entire high temperature flow field as a function of time. The time-averaged temperature field for each phase of the pulsing methanol fire was also determined. The results provide insight into the dynamic character of turbulent pool fires.
\end{abstract}

KEYWORDS: methanol; pool fires, pyrometry; temperature field measurements. 


\section{BACKGROUND}

The focus of this study was the development of a new data set characterizing the time-varying temperature field in a medium-scale pool fire steadily-burning in a well-ventilated, quiescent environment. Pool fires are a fundamental type of combustion phenomena in which the fuel surface is isothermal, flat, and horizontal, which provides a simple and well-defined configuration for testing models and furthering the understanding of fire phenomena. The fires are characterized by a dominant puffing frequency associated with the large-scale vortical structures generated by the fire [1]. In this study, methyl alcohol (methanol; $\mathrm{CH}_{3} \mathrm{OH}$ ) was selected as the fuel for investigation since it yields a fire in which no carbonaceous soot is present.

Use of fire modeling in fire protection engineering has dramatically increased during the last decade due to the development of practical computational fluid dynamic fire models and the decreased cost of computational power. Today, fire protection engineers use models like the Consolidated Fire and Smoke Transport Model (CFAST) and the Fire Dynamics Simulator (FDS) to design safer buildings, nuclear power plants, aircraft cabins, trains, and marine vessels to name a few types of applications $[2,3]$. To be reliable, the models require validation, which involves a large collection of experimental measurements. Traditionally, model validation studies in fire research are typically conducted through comparison of model results with time-averaged and root mean square (rms) measurements of quantities such as temperature and heat flux. The objective of this study is to provide a data set of time-varying field measurements from a medium-scale pool fire.

Bryant [4] reported on the measured velocity field in a compartment doorway using stereoscopic particle imaging velocimetry. A method based on thin filament pyrometry has been previously used to analyze temperature behavior in time varying, non-premixed flames. Hariharan et al. measured the structure of a blue fire whirl burning heptane and other hydrocarbons using fine wire thermocouples and thin-filament pyrometry [5,6]. Pitts used thin filament pyrometry to characterize the structure of acoustically phase-locked flickering methane-air diffusion flames [7]. Most fire studies typically report time-averaged measurements - even if many simultaneous local measurements were conducted (e.g., Ref. [8]). Furthermore, studies in the fire literature commonly use large diameter thermocouples (>500 $\mu \mathrm{m}$ ), which have relatively large time constants (> $1 \mathrm{~s}$ ), which results in instrument response times that are not particularly useful for understanding the time-resolved structure of time varying fires.

Fires burning methanol are particularly unusual since no carbonaceous soot is present. The absence of soot creates conditions advantageous for application of thin filament pyrometry methods as there is no background blackbody radiation source, which otherwise would compete with luminosity from the thin filament. Table 1 provides a list of previous measurements in a well-ventilated, $30 \mathrm{~cm}$ diameter methanol pool fire steadily-burning in a quiescent environment, which are complementary to the measurements reported here. Weckman and Strong [9] made point measurements of the local velocity and temperature fields up to one diameter above the fuel surface of the methanol pool fire for conditions similar to those considered here - they used a $30.5 \mathrm{~cm}$ diameter burner with a lip height of $1 \mathrm{~cm}$. Ref. [10] reproduced a portion of the gas-phase temperature data reported in Ref. [9] and extended the spatial extent of the data to $60 \mathrm{~cm}$ above the pool surface. The radiative heat flux 
distribution both onto the fuel surface and emitted to the surroundings for the $0.30 \mathrm{~m}$ methanol pool fire are discussed in Refs. [11, 12,13]. Global flame parameters such as the mean flame height and pool fire pulsation frequency have also been characterized $[9,12]$.

Table 1. Previous Measurements in a Well-Ventilated, Steadily-Burning, $30 \mathrm{~cm}$ Diameter, Methanol Pool Fire.

\begin{tabular}{|l|c|}
\hline Parameter & Reference \\
\hline Mean flame height $(\mathrm{m})$ & 9 \\
\hline Pulsation frequency $(\mathrm{Hz})$ & 9 \\
\hline Radiative fraction $\left(\mathrm{kW} / \mathrm{m}^{2}\right)$ & $9,11,12,13,14$ \\
\hline Mass loss rate $(\mathrm{g} / \mathrm{s})$ & $9,11,15$ \\
\hline Heat Release Rate $(\mathrm{kW})$ & 11 \\
\hline Radiative flux distribution onto fuel surface $\left(\mathrm{kW} / \mathrm{m}^{2}\right)$ & 12 \\
\hline Total heat flux distribution onto fuel surface $\left(\mathrm{kW} / \mathrm{m}^{2}\right)$ & 11 \\
\hline Radiative flux distribution to surroundings $\left(\mathrm{kW} / \mathrm{m}^{2}\right)$ & 11 \\
\hline Vertical temperature distribution in the fuel $\left({ }^{\circ} \mathrm{C}\right)$ & 12 \\
\hline Gas species volume fraction $(\mathrm{mol} / \mathrm{mol})$ & 10 \\
\hline Temperature Field $\left({ }^{\circ} \mathrm{C}\right)$ & 9,15 \\
\hline Velocity Field $(\mathrm{m} / \mathrm{s})$ & 9 \\
\hline
\end{tabular}

The table above lists both local and global measurements characterizing the structure of the $0.30 \mathrm{~m}$ diameter methanol pool fires from many previous investigations [9 - 15]. The measurements reported here complement the measurements previously reported in these investigations with the objective of building a broad picture of the energetics, structure, and dynamics of this type of fire.

This study presents measurements characterizing the transient temperature field in a medium-sized pool fire burning methanol. The results are presented in terms of both the time-averaged field temperature and as the field temperature representative of each of the various phases that characterize the evolving fire as it undergoes its puffing cycle. This report is divided into several parts. In Section 2, the experimental method and apparatus are described. The results are discussed in Section 3 and summarized in Section 4. Acknowledgements and references are provided in Sections 5 and 6, respectively.

\section{EXPERIMENTAL METHOD}

Steady-state burning conditions were established before measurements were initiated. A warm-up period of 10 min was required to achieve a steady burning rate. Since back diffusion of water slowly accumulates in the fuel pool in methanol fires, fresh fuel was used between experiments. Experiments were conducted under an exhaust hood located $2 \mathrm{~m}$ above the burner rim. The experimental apparatus and methods have been previously described in Refs. [10,11]. 


\subsection{Exhaust Hood and Compartment}

The fuel pan was placed in a compartment with dimensions as indicated in Figure 2. The walls of the compartment $(2.5 \mathrm{~m}$ by $2.5 \mathrm{~m})$ were formed by a double layer of wire mesh screen $(5 \mathrm{mesh} / \mathrm{cm})$, which created a porous barrier that reduced the influence of room currents on the flow field. The bottom of the exhaust hood $(1.8 \mathrm{~m}$ by $1.8 \mathrm{~m}$ ) was positioned $2.1 \mathrm{~m}$ above the floor, whereas the exhaust inlet $(1.5 \mathrm{~m}$ by $1.5 \mathrm{~m}$ ) was positioned about $2.6 \mathrm{~m}$ above the floor. The exhaust flow was maintained at $0.50 \mathrm{~kg} / \mathrm{s}$ as measured by a system of bidirectional probes and averaging Pitot tubes.

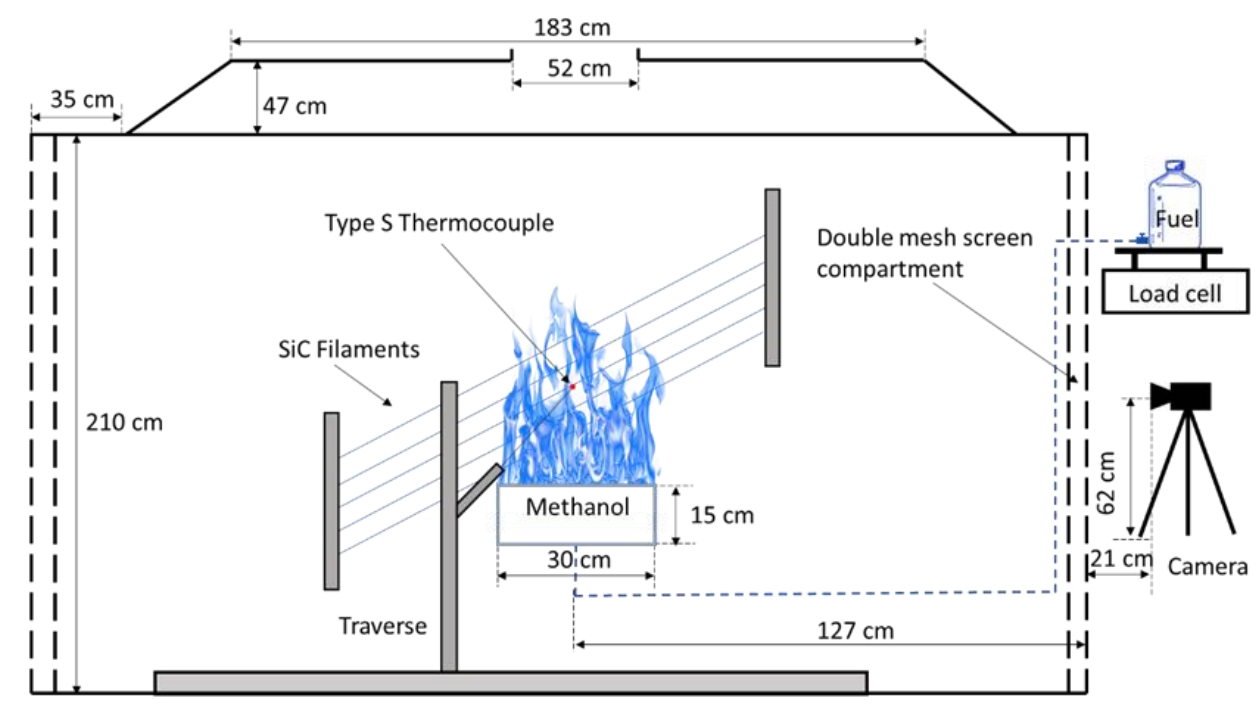

Figure 1. Schematic drawing of the compartment with double wire mesh screen. A traverse was used to move the thermocouple. In a separate experiment, an array of 23 filaments was mounted above the pol burner.

\subsection{Pool Burner}

A circular stainless-steel pan with an inner diameter (D) of $0.30 \mathrm{~m}$, a depth of $0.15 \mathrm{~m}$, and a wall thickness of $0.0013 \mathrm{~m}$ was used to hold the liquid fuel. The bottom of the burner had a water-cooled section with thermocouples monitoring the water temperature. The water flow rate was about $1 \mathrm{~L} / \mathrm{min}$, ensuring that the bottom of the burner was maintained at a near constant temperature $\left(20^{\circ} \mathrm{C} \pm 3{ }^{\circ} \mathrm{C}\right) .{ }^{1}$ The burner was mounted on $7.7 \mathrm{~cm}$ "legs" such that the top of the burner rim was $0.27 \mathrm{~m}$ above the floor. A fuel overflow section, included for safety, was positioned at a location $10 \mathrm{~cm}$ below the rim; it extended $2.5 \mathrm{~cm}$ in the radial direction beyond the fuel reservoir outer wall.

Fuel to the burner was gravity fed from a reservoir on a load cell monitored by a data acquisition system. The fuel level was verified throughout the experiment by visually observing the tiny tip of a sharpened ( $2 \mathrm{~mm}$ diameter) pointer that formed a barely discernable bulge on the fuel surface. The level of the fuel was maintained $10 \mathrm{~mm}$ below the burner rim by regulating the fuel supply from the reservoir to the burner, using either a thermocouple located just above the pool surface to control a solenoid valve in the

\footnotetext{
${ }^{1}$ Unless otherwise noted, the uncertainty in this paper is expressed as the combined uncertainty with a coverage factor of two, representing a $95 \%$ confidence interval.
} 
fuel feed line or by manually adjusting a valve to control the rate of fuel flow. Based on observations, the expanded uncertainty in the fuel level was estimated to be $1 \mathrm{~mm}$.

\subsection{SiC Filaments}

An array of $14 \mu \mathrm{m}$ diameter Silicon-Carbide (SiC) filaments (Nicalon Type NL 200) ${ }^{2}$ were oriented horizontally at various heights above a central cross-section of the $0.30 \mathrm{~m}$ diameter methanol pool fire. The filaments were packaged in a bundle of 500 units encased in a polyvinyl acetate coating. Baking the bundle for 4 hours at $350{ }^{\circ} \mathrm{C}$ removed the coating, allowing separation of the individual filaments from the bundle. The filaments were cut to approximately $0.85 \mathrm{~m}$ and each end was attached to string using a fast-drying cyanoacrylate liquid adhesive. The assembly was stretched between two pieces of an extruded aluminum structural framing system that were separated by approximately $0.89 \mathrm{~m}$. Twenty-three filaments were aligned in a plane, parallel to each other, one above the other. The lowest filament was $0.6 \mathrm{~cm}$ above the burner, while the highest was $0.60 \mathrm{~m}$ above. The distance between the filaments varied with height above the burner as shown in Figure 2. Figure 3 is a photo of the experimental apparatus. A literature review suggests that this study employed the longest filament lengths and the largest number of filaments simultaneously used for instantaneous thin filament pyrometry measurements.

The composition and characteristics of the filaments have been described by Ichikawa [16]. For typical flame conditions, the filament time response has been estimated as about $1 \mathrm{~ms}$ [17, 18]. Heat conduction along the filament impacts the spatial resolution of the pyrometry method, which has been estimated to be on the order of $100 \mu \mathrm{m}[17,18]$.

The local gas-phase temperature was determined from the measured filament intensity. The filament intensity as a function of location was determined using the grayscale values of the sum of seven pixels perpendicular to the filament following Ref. [5]. Calibration of the thin filament pyrometry measurement is described below.

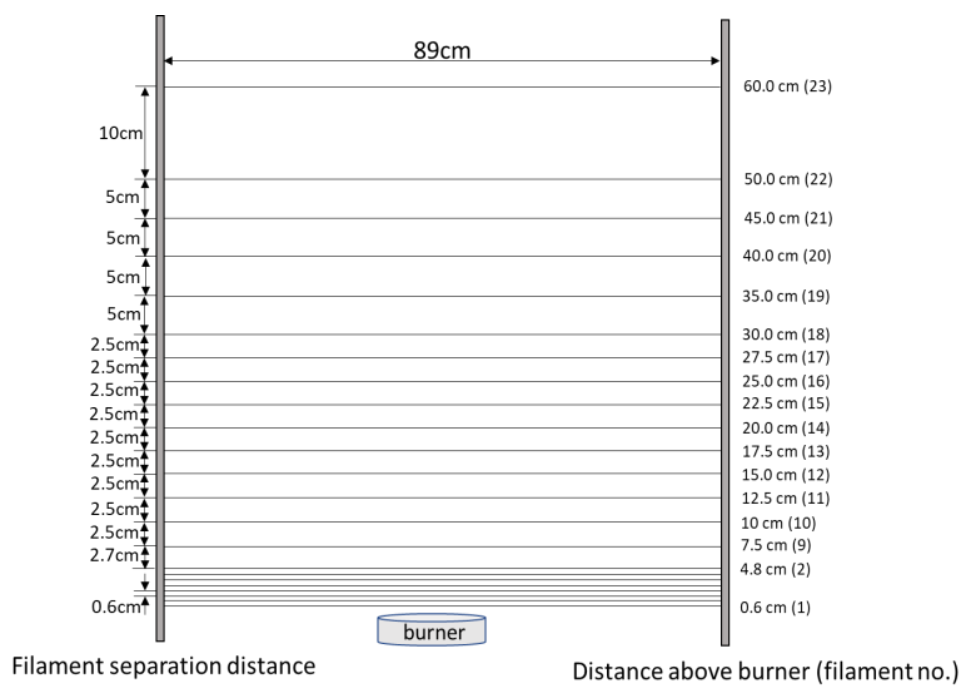

Figure 2. Arrangement of the SiC filaments above the burner.

\footnotetext{
${ }^{2}$ Certain commercial entities, equipment, or materials may be identified in this document in order to describe an experimental procedure or concept adequately. Such identification is not intended to imply recommendation or endorsement by the National Institute of Standards and Technology, nor is it intended to imply that the entities, materials, or equipment are necessarily the best available for the purpose.
} 


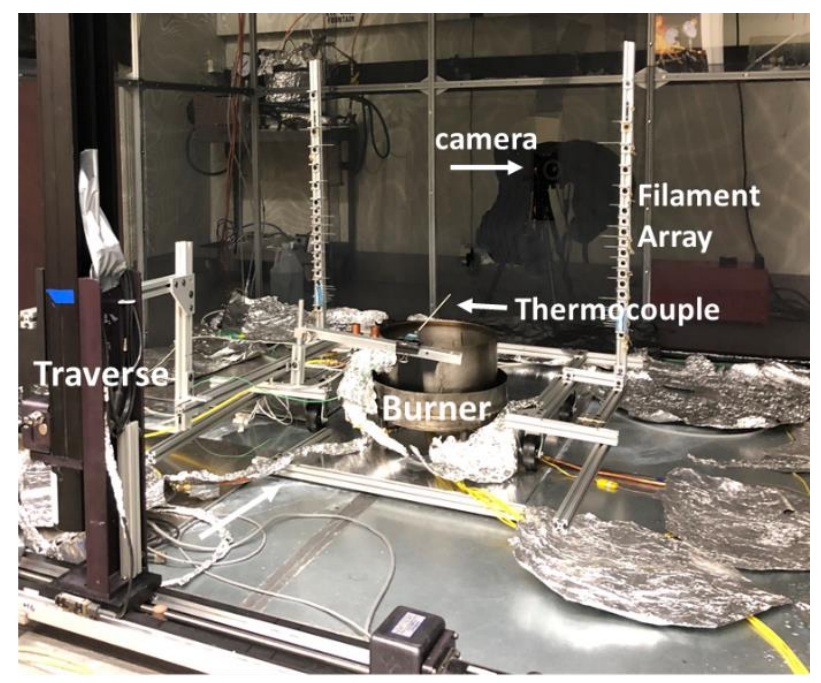

Figure 3. Experimental apparatus.

\subsection{Thermocouple Temperature Measurement}

A $50 \mu \mathrm{m}$ diameter bare-bead Type $\mathrm{S}(\mathrm{Pt}-10 \% \mathrm{Rh} / \mathrm{Pt}$ ) thermocouple mounted on a $20 \mathrm{~cm}$ long, $3 \mathrm{~mm}$ outer diameter, ceramic tube with two holes was used to calibrate the thin filament pyrometry measurement. The thermocouple and filament measurements were not conducted at the same time. Thermocouple calibration measurements were made at 7 radial positions about the center of the flame at a height of $10 \mathrm{~cm}$ above the burner. The thermocouple time response was estimated as $0.02 \mathrm{~s}$ [19]. The thermocouple leads were oriented horizontally in the fire. The time series of the thermocouple signal was acquired at a rate of $60 \mathrm{~Hz}$ for $90 \mathrm{~s}$ at 7 locations near the fire centerline of the fire $10 \mathrm{~cm}$ above the burner surface. The inherent accuracy of Type $\mathrm{S}$ thermocouples is $1.5 \mathrm{~K}$ or $0.25 \%$, whichever is larger [20].

\subsubsection{Pyrometry Calibration Strategy}

The calibration strategy employed the notion that at each flame location, the thermocouple and the filament experienced the same thermal conditions. This implies that the probability density functions (PDF) of the thermocouple signal and the filament signal were positively correlated. By considering the PDFs, a calibration curve relating the thermocouple temperature and the filament intensity was obtained. For the sake of calibration, the PDFs of filament intensity and thermocouple temperature were broken into 100 parts, with the average of each of the parts used to construct the calibration curve. Since the filament intensity signal was only observable when the local temperature was sufficiently high, flame locations with the highest temperatures were selected in construction of the calibration. The high temperature locations were identified based on previously reported thermocouple measurements reported in this fire $[9,10]$.

\subsubsection{Thermocouple Radiation Correction}

The thermocouple measurements were corrected for radiative losses considering an energy balance at the thermocouple bead [21]. The convective- radiative energy balance can be written as:

$$
\left(T-T_{m}\right) N u \frac{k}{D}=\sigma \varepsilon\left(T_{m}{ }^{4}-T_{a}{ }^{4}\right)
$$


where $T$ is the gas temperature, $T_{m}$ is the temperature at the thermocouple bead, $T_{a}$ is the ambient temperature $(298 \mathrm{~K}), k$ is the gas thermal conductivity, $D$ is the diameter of the thermocouple bead, $\sigma$ is the Stefan-Boltzmann constant, $\varepsilon$ is the thermocouple emissivity, and $N u$ is the Nusselt number. Since soot was not present in the methanol fire, the thermocouple emissivity and diameter were considered invariant with time. The bead was approximately spherical as seen in an optical microscope with the bead diameter approximately two times the diameter of the lead wires. The Nusselt number for a sphere is given by Ref. [21]:

$$
N u=2+0.6 \operatorname{Re}^{1 / 2} \operatorname{Pr}^{1 / 3}
$$

where $R e$ is the Reynolds number and $P r$ is the Prandtl number. The temperature dependent gas properties for $\mathrm{k}, \operatorname{Re}$ and $\operatorname{Pr}$ were taken as those of air and the temperature dependent platinum emissivity was taken from Ref. [21]. To determine the $R e$, the measured gas velocity in a $30 \mathrm{~cm}$ methanol fire was taken from Ref. [9]. Solving Eq. 1 for $T$, a radiative correction for the gas temperature was found to be less than $30 \mathrm{~K}$ for the peak fire temperature, which was approximately $1900 \mathrm{~K}$. The sensitivity of the value of the temperature correction to uncertainty in the velocity was less than $2 \mathrm{~K}$.

\subsection{Camera}

A Nikon 500D single lens reflex digital camera fitted with optical filters and a zoom lens was used to record the emission intensity of the filaments. The camera was positioned on a tripod and focused onto the center of the filament array, which was located $1.41 \mathrm{~m}$ from the camera. Setting the zoom lens to $35 \mathrm{~mm}$ allowed imaging of the entire $0.3 \mathrm{~m}$ by $0.6 \mathrm{~m}$ high temperature flow field. The aperture size and gain levels were adjusted to avoid pixel saturation. The camera was fitted with a BG7 blue bandpass optical filter and a $10 \%$ neutral density filter. The camera's aperture and gain settings were adjusted to prevent pixel saturation. The camera F number was set to 2.8 , the ISO speed was 2000, and the shutter speed was $1 / 60 \mathrm{~s}$. The maximum grayscale pixel intensity was 8 bit in Red, Green, and Blue. The red, green, and blue (RGB) pixel intensities were extracted from the images and converted to grayscale as a weighted average of RGB values equal to $(0.2989 \mathrm{R}+0.5870 \mathrm{G}+0.1140 \mathrm{~B})$ following Ref. [5]. Experiments collected $120 \mathrm{~s}$ of $29.97 \mathrm{~Hz}$ video at 4K Ultra High Definition (a frame size of $2840 \mathrm{x} 2160$ pixels) and 3400 frames were used in the analysis. The results were multiplied by $2^{8}$ as part of the calculation procedure such that the maximum pixel intensity was $65535\left(2^{16}-1\right)$. Analogous experiments were conducted with the camera set to tagged image file format (TIFF) rather than video mode. About 1000 images were collected in 12 bit TIFF mode at the camera's maximum frame rate of $10 \mathrm{~Hz}$. All of the camera images were analyzed using Mathlab software.

\section{RESULTS AND DISCUSSION}

\subsection{Thermocouple Temperature and Filament Intensity}

Figure 4 shows snapshots of the pulsing methanol fire burning in the $0.30 \mathrm{~m}$ stainless-steel water-cooled burner with the thin filaments mounted above the central plane of the burner. Some portions of the images show blue methanol flames in the presence of glowing filaments; other portions of the image show blue flames without the presence of glowing filaments, suggesting that the flames were away from the plane defined by the filament array. This low Froude number fire had a narrow necking region and 
was characterized by a prominent puffing cycle in which a series of repeated large vortical structures were observed to roll towards the fire centerline and neck-in to form a narrow and long visible fire plume. Curved flame sheets were anchored at the burner rim forming the base of the fire. The dynamic fire shape was consistent with previous descriptions of a medium-scale pool fire [1,22].
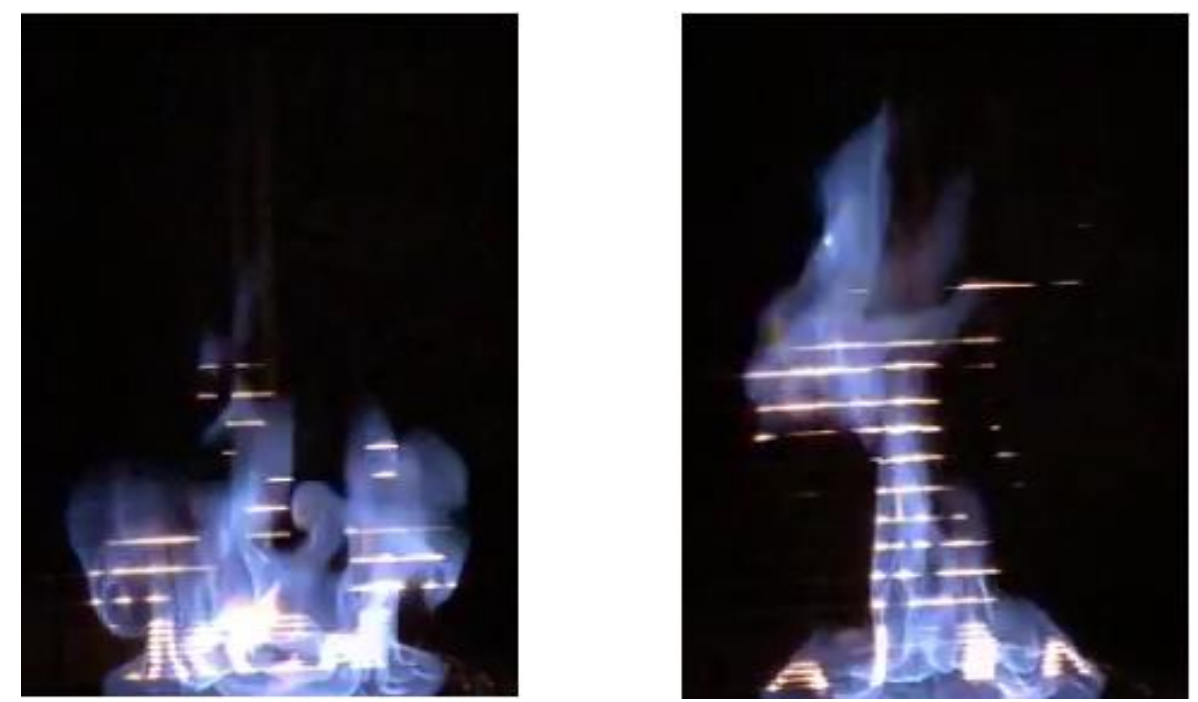

Figure 4. Two images of the glowing filaments mounted above the central plane of the methanol pool fire.

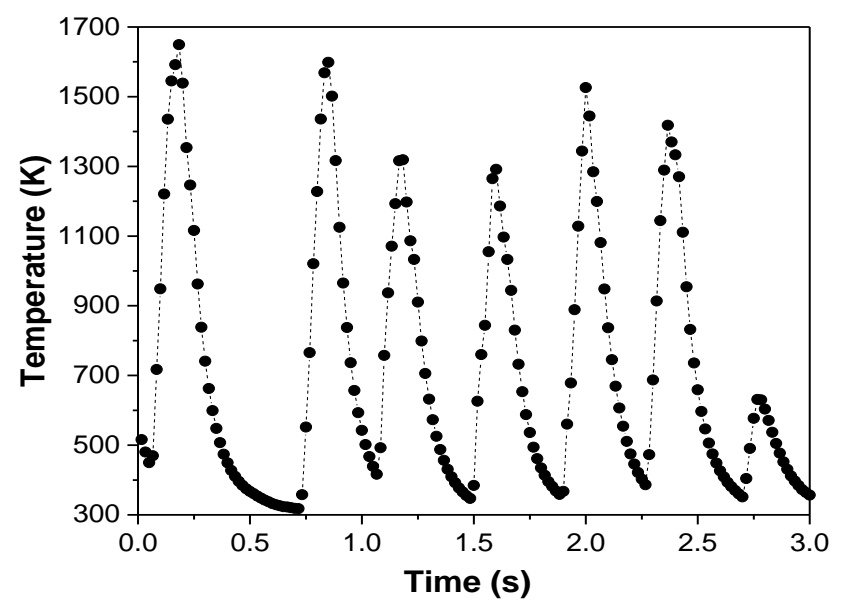

Figure 5. Typical $3 \mathrm{~s}$ time series of the radiation-corrected thermocouple temperature measurement at $(\mathrm{Z}, \mathrm{R})=$ $(10 \mathrm{~cm}, 12 \mathrm{~cm})$. The combined uncertainty was less than the size of the symbols, equivalent to $\pm 13 \mathrm{~K}$ at $1700 \mathrm{~K}$.

Figure 5 shows a typical trace of the local, radiation-corrected, thermocouple temperature as a function of time in the steadily burning methanol pool fire. The data was acquired at a sampling rate of $60 \mathrm{~Hz}$ at a position $(Z, R)=(10 \mathrm{~cm}, 12 \mathrm{~cm})$, where $\mathrm{Z}$ and $\mathrm{R}$ represent the distance above the burner and the distance from the burner centerline, respectively. This position experiences large intermittent extremes of 
temperature from $300 \mathrm{~K}$ to nearly $1700 \mathrm{~K}$. The temperature appeared to be periodic as discussed below. The radiation-corrected temperatures had an estimated combined uncertainty equal to about $\pm 13 \mathrm{~K}$ at $1700 \mathrm{~K}$ due to uncertainty in the calibration and the radiation correction. This value is smaller than the symbols in the figure.

A typical trace of the video-mode grayscale filament intensity at the same fire location is shown in Figure 6. A large fraction of the grayscale time series had values of zero intensity. At this location, $80 \%$ of the grayscale intensities were equal to 0 , while $20 \%$ of the instances had grayscale intensities with non-zero values or a fractional probability, $\mathrm{P}$, of 0.20 . The range of $\mathrm{P}$ varied from 0 to 0.99 , depending on location as seen in Figure 7. The largest values tended to be close to the fuel surface and along the fire centerline.

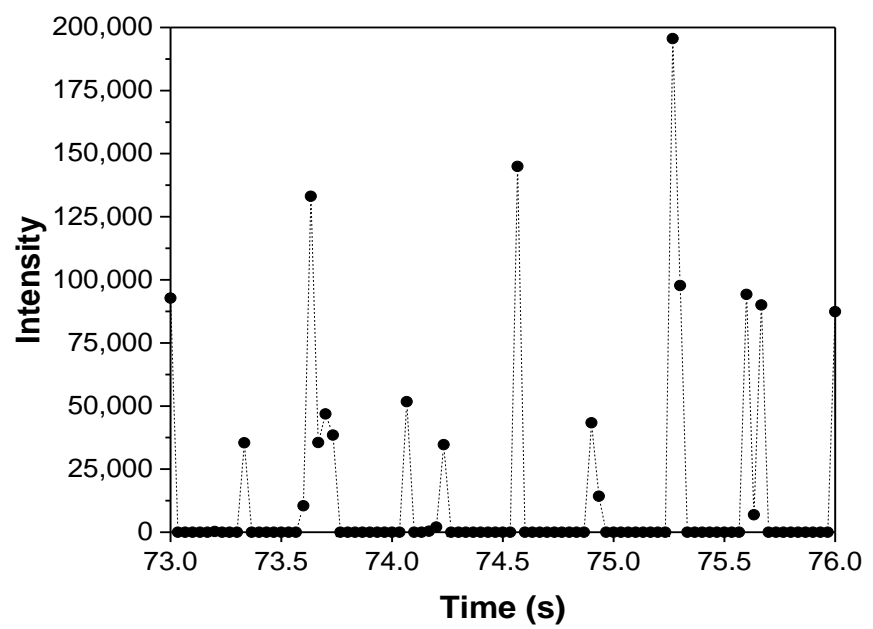

Figure 6. Typical $3 \mathrm{~s}$ time series of filament video-mode grayscale intensity at $(\mathrm{Z}, \mathrm{R})=(10 \mathrm{~cm}, 12 \mathrm{~cm})$ acquired at a sampling rate of $30 \mathrm{~Hz}$.

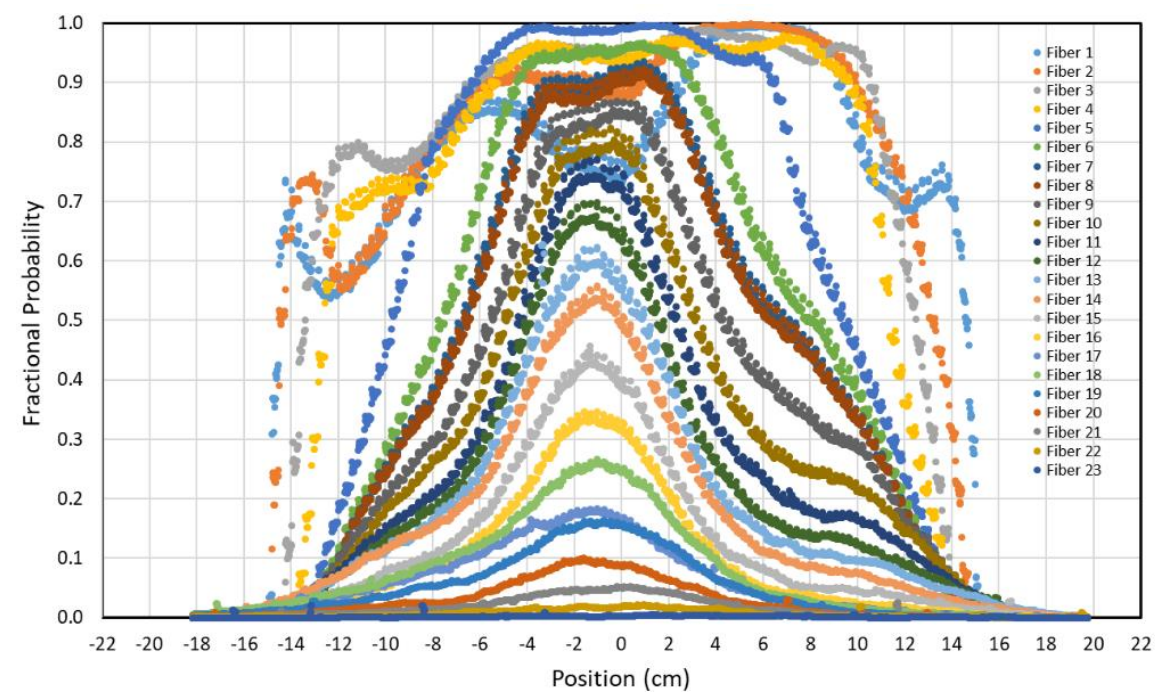

Figure 7. The probability of non-zero grayscale intensity as a function of location on each of the filaments. 
Measurements like those in Figure 5 and Figure 6 were used to develop a temperature-luminosity calibration, considering multiple locations with large $\mathrm{P}$ values. Figure 8 shows the relationship between the mean, radiation-corrected, thermocouple temperature and filament video-mode grayscale pixel intensity measurements averaged at seven fire locations. The measurement variance is also shown, which decreased with temperature, and was equal to $86 \mathrm{~K}$ at $1000 \mathrm{~K}$ and $15 \mathrm{~K}$ at $1800 \mathrm{~K}$.

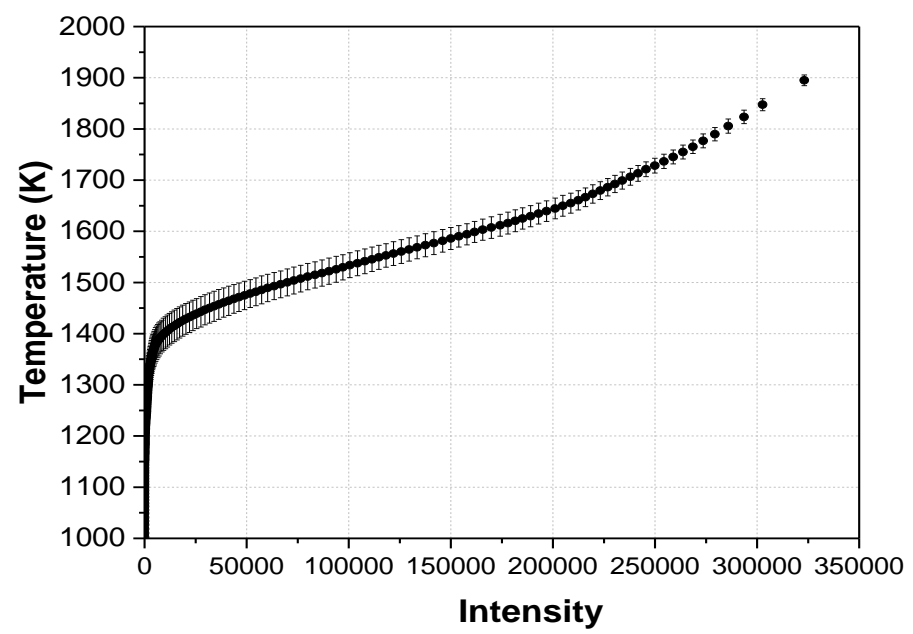

Figure 8. The averaged radiation-corrected thermocouple temperature and grayscale video-mode filament intensity. The variance was determined from measurements at seven fire locations.

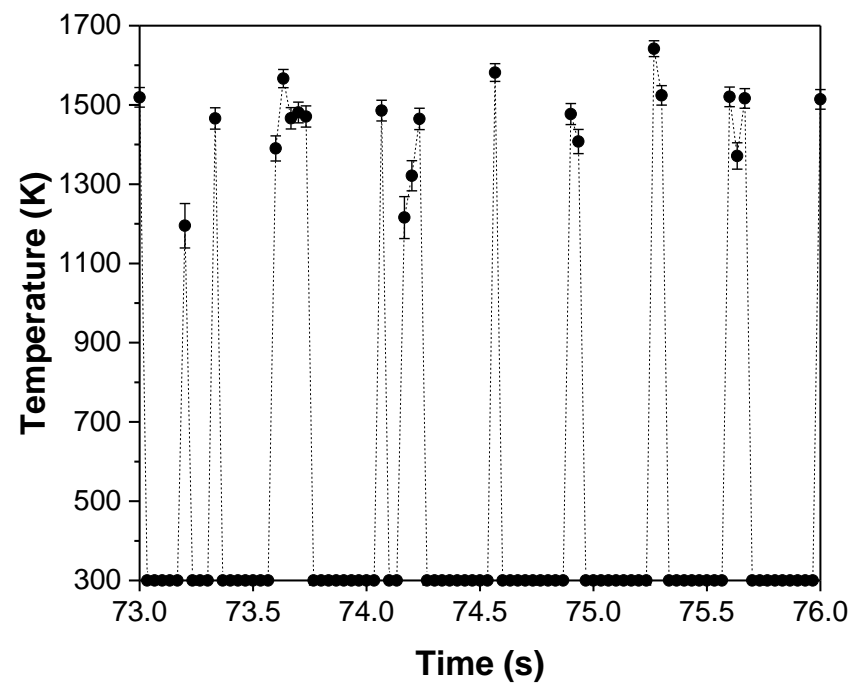

Figure 9. Time series of temperature at $(Z, R)=(10 \mathrm{~cm}, 12 \mathrm{~cm})$ corresponding to the data shown in Figure 6 with the zero intensity instances assumed to equal $300 \mathrm{~K}$. The combined uncertainty is also shown. 

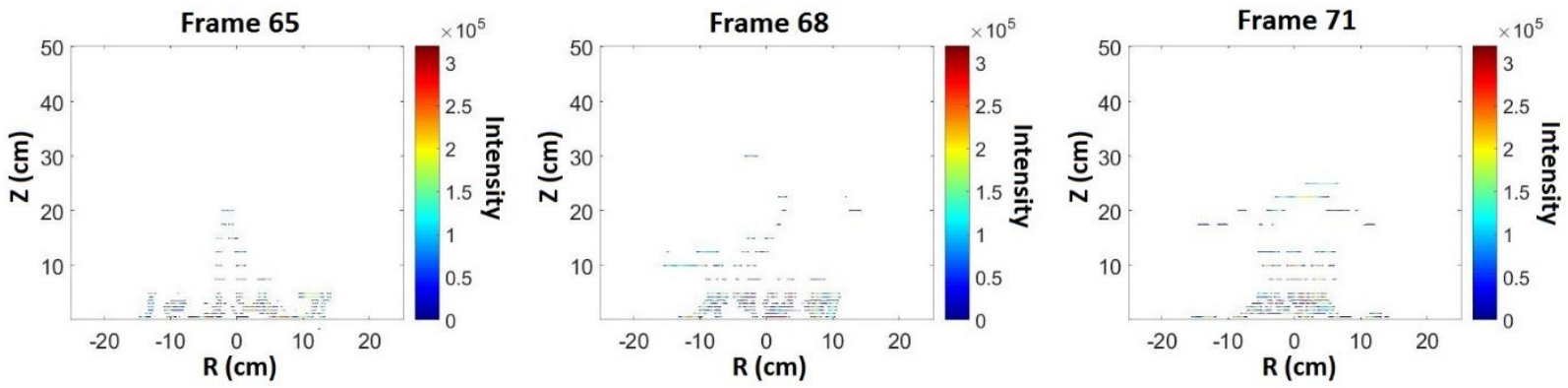

Figure 10. False color representation of the filament grayscale intensities in the flow field at three instances in time, each separated by $0.1 \mathrm{~s}$, in the pulsing methanol fire. Here, $\mathrm{Z}$ and $\mathrm{R}$ represent the distance above the burner and distance from the burner centerline, respectively.

Using the calibration, Figure 9 replots the data shown in Figure 6, showing the transient local temperature at $(Z, R)=(10 \mathrm{~cm}, 12 \mathrm{~cm})$. The figure also shows the combined measurement uncertainty, which was dominated by the uncertainty in the calibrations (see Figure 8).

Figure 10 shows the instantaneous filament grayscale intensity field at three instances in time separated by approximately $0.1 \mathrm{~s}$. The figure shows the changing intensity field, which exhibits substantial changes in its shape and the values of the resulting temperature.

Figure 11 shows false color maps of the instantaneous field temperature which were interpolated from the instantaneous filament grayscale measurements (like those in Figure 10) after applying the calibration curve (Figure 8).
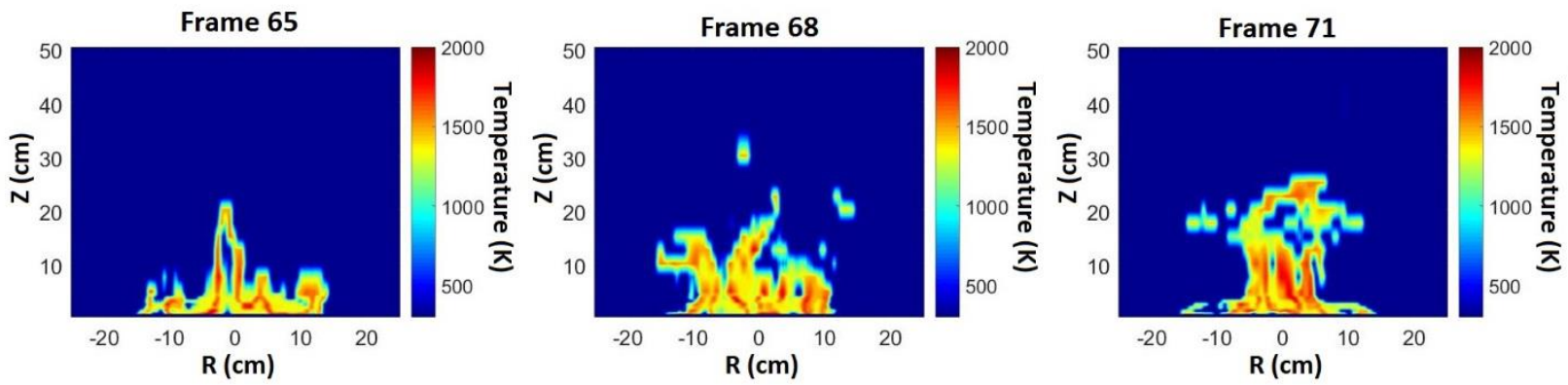

Figure 11. Typical instantaneous field measurements of temperature (determined from the filament intensity) at three instances in time separated by about $0.1 \mathrm{~s}$ in the pulsing methanol fire. Here, $\mathrm{Z}$ and $\mathrm{R}$ represent the distance above the burner and distance from the burner centerline, respectively.

Image analysis of the video record of the instantaneous filament luminosity (such as seen in Figure 10) was used to quantify the fire's puffing (or pulsation) frequency. Figure 12 shows the results of an analysis of 300 fire pulsation cycles for which the highest probability of cyclic periodicity was 11 frames/cycle. The average period was found to equal $11.3( \pm 0.3)$ frames/cycle (or $0.38 \mathrm{~s} \pm 0.01 \mathrm{~s}$ ), which corresponds to a frequency of $2.65 \mathrm{~Hz} \pm 0.08 \mathrm{~Hz}$. The results were consistent with Fourier power spectra of the thermocouple temperature and the filament luminosity time series in the highly intermittent region $(\mathrm{Z}=10 \mathrm{~cm}, \mathrm{R}=12 \mathrm{~cm}$; see Figure 5 and Figure 6) yield the same dominant frequency equal to $2.64 \mathrm{~Hz} \pm 0.06 \mathrm{~Hz}$ and $2.64 \mathrm{~Hz} \pm 0.03 \mathrm{~Hz}$, respectively. These results are consistent with previous results [23]. 


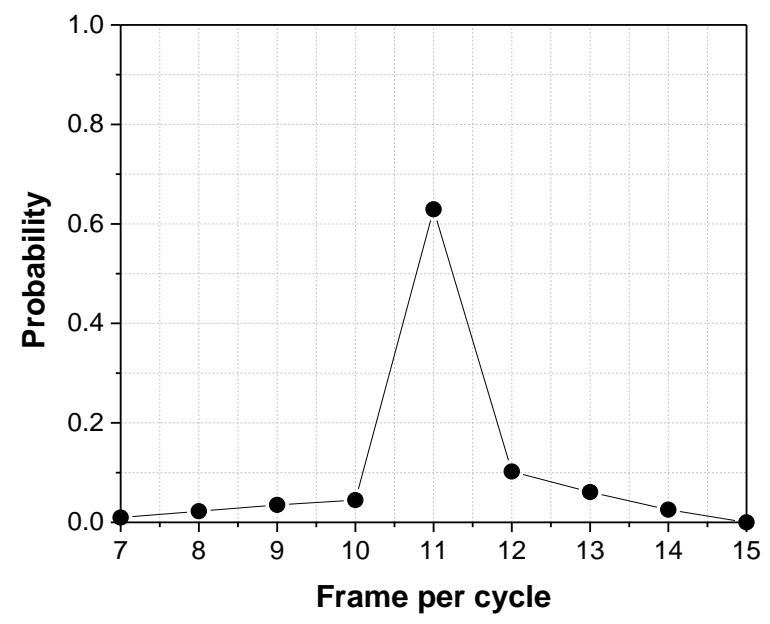

Figure 12. Normalized probability distribution of the number of $30 \mathrm{~Hz}$ video frames for one fire pulsation cycle.

\subsection{Estimate of the Temperature Field}

The mean temperature field was determined from the filament intensity measurements using three approaches. First, the mean values of the high-temperature filament results were calculated as an interpolated, false color, temperature map as seen in Figure 13. For locations where $\mathrm{P} \approx 1$ (see Figure 7), the results in Figure 13 were expected to be a reasonable estimate of the average field temperature. For locations where $\mathrm{P}<<1$, the results in Figure 13 were expected to have a positive bias since the "cold" filament instances were not considered in the calculation.

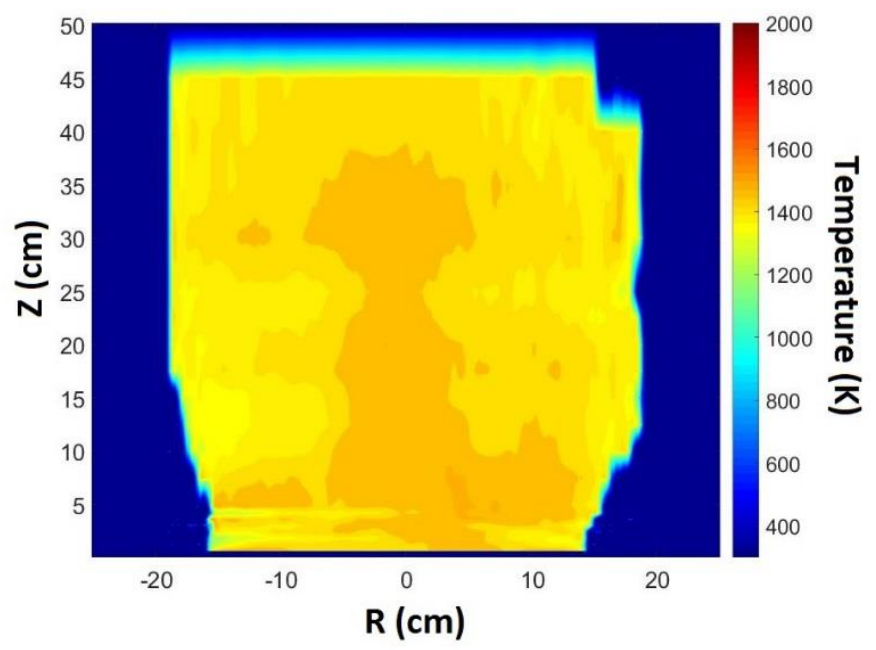

Figure 13. False color map of the mean high temperature filament measurements as a function of location. Here, $\mathrm{Z}$ and $\mathrm{R}$ represent the distance above the burner and distance from the burner centerline, respectively.

Figure 14 compares the time-averaged, high temperature, filament data (like Figure 13 to previously reported thermocouple measurements [9] as a function of distance from the burner centerline for locations $10 \mathrm{~cm}$ above the burner. As expected, consideration of only the high temperature filament data led to a 
positive bias of the time-averaged mean local temperature compared to the thermocouple measurements with the differences especially significant near the edges of the fire when $\mathrm{P}<<1$.

The second approach shown in the figure was denoted the "cold filament" approximation in which the temperature was assumed to equal $300 \mathrm{~K}$ when the filament luminosity was too small to measure. As expected, this assumption led to a negative bias of the estimated time-averaged local temperature as compared to the previously reported thermocouple measurements [9].

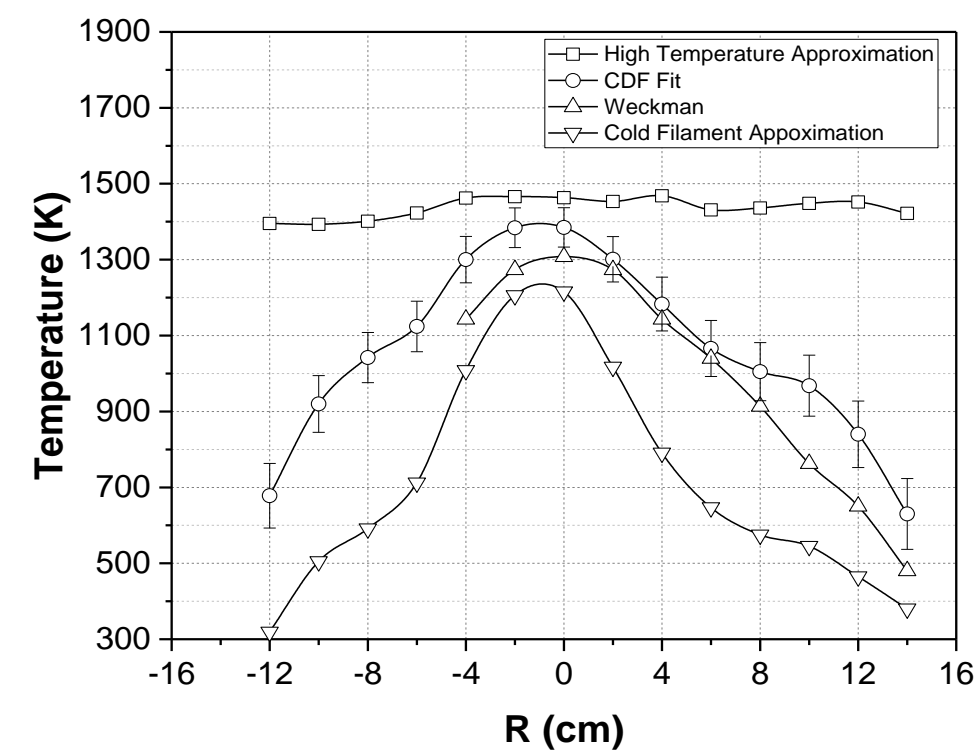

Figure 14. The time-averaged temperature profiles $10 \mathrm{~cm}$ above the burner surface compared to thermocouple measurements reported by Weckman and Strong [9].

The third approach shown in the figure, denoted "Gaussian (CDF)", estimated the mean temperature in the time series from a fit of the cumulative density function (CDF) to a Gaussian distribution,

$\mathrm{G}\left(T_{i} \mid \mu, \sigma\right)$, for all flame locations. A regression analysis of the CDF was used to estimate the mean and standard deviation for the entire field. The CDF was given by:

$$
\mathrm{G}\left(T_{i} \mid \mu, \sigma\right)=\frac{1}{2}\left[1+\operatorname{erf}\left(\frac{T_{i}-\mu}{\sigma \sqrt{2}}\right)\right]
$$

where $T_{i}$ is the $\mathrm{i}^{\text {th }}$ temperature determination, $\mu$ is the mean and $\sigma$ is the standard deviation of the distribution. The fit to the cumulative density function at each location in the fire was obtained even when $\mathrm{P}<<1$. The expanded uncertainty to the regression analysis is shown as error bars in the figure. The combined uncertainty, which was larger, was dominated by the uncertainty in the calibration and varied as a function of temperature. At $1000 \mathrm{~K}$, the combined uncertainty was equal to $\pm 200 \mathrm{~K}$. Figure 14 shows that the trends in the CDF results were in general agreement with the trends in the thermocouple measurements reported by Weckman [9]. Thermocouple measurements made as part of this study are also shown in the figure. For $\mathrm{P}>0.5$, the TIFF mode temperature results were on-average within $34 \mathrm{~K}$ of the video temperature results, which was within experimental uncertainty. The rest of this paper focuses on the video results. 
Figure 15 shows typical measurements and fitted Gaussian distributions plotted as CDFs for two fire locations $(Z, R)=(10 \mathrm{~cm}, 12 \mathrm{~cm})$ and $(10 \mathrm{~cm}, 0 \mathrm{~cm})$, where $\mathrm{P}$ was equal to 0.14 and 0.77 , respectively. From the 3400 camera frames, only 476 frames were available to compose the CDF since $\mathrm{P}$ was equal to 0.14. As exemplified in the figure, the goodness of fit was generally better when $\mathrm{P}$ was larger. The value of the coefficient of determination $\left(\mathrm{R}^{2}\right)$ was always greater than about 0.9 , which characterized the efficacy of using a Gaussian distribution.
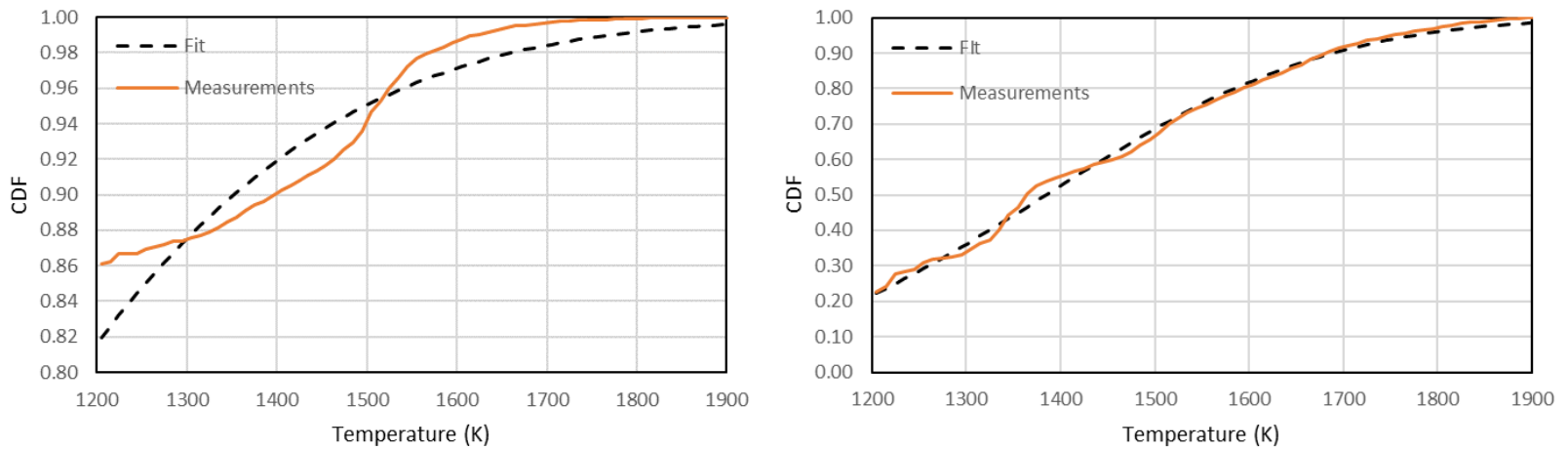

Figure 15. CDF measurements and fits for positions $(\mathrm{Z}=10 \mathrm{~cm}, \mathrm{R}=12 \mathrm{~cm}$; left $)$ and $(\mathrm{Z}=10 \mathrm{~cm}, \mathrm{R}=0 \mathrm{~cm}$; right).

Figure 16 shows the normalized expanded uncertainty (95\% confidence level) in the CDF fit for many fire locations as a function of the mean temperature, which was estimated following Ref. [24]. As the temperature decreased, $\mathrm{P}$ decreased, and the uncertainty increased. For the largest temperatures, the expanded uncertainty was about $6 \%$ of the mean temperature, which was about a factor of 7 larger than the inherent thermocouple uncertainty. For the smallest temperatures, the normalized expanded uncertainty approached $30 \%$.

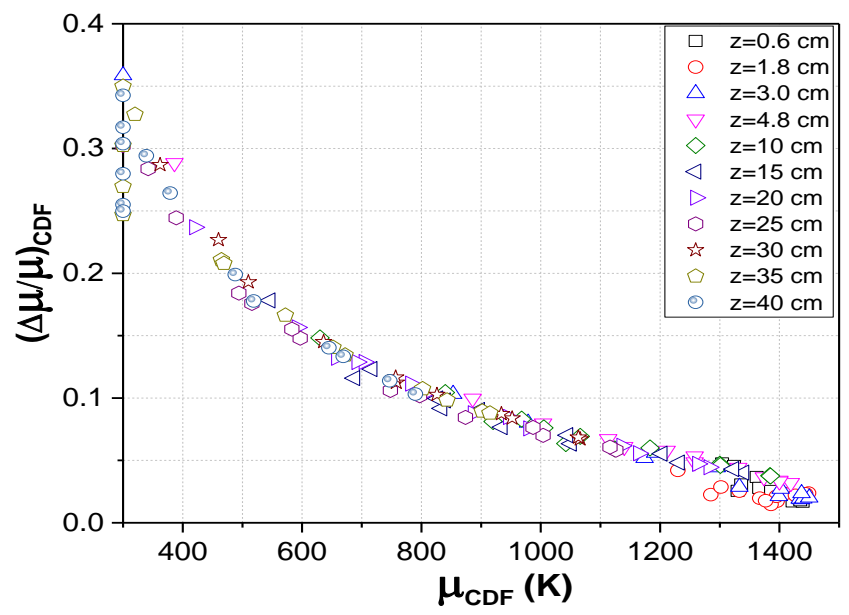

Figure 16. Relationship between the normalized uncertainty in the temperature determined using CDF fits for various radial positions on 11 filaments.

Figure 17 shows a false color map of the time-averaged temperature field determined using the CDF method. The largest mean temperatures were found just above the burner surface as expected from previous measurements $[9,10]$. 
Figure 18 shows the time-averaged temperature field calculated using the CDF fit method compared to the mean temperature determined using the cold filament approximation method (which set the zero grayscale filament intensity instances equal to $300 \mathrm{~K}$ ). The results were approximately represented by a $6^{\text {th }}$ order polynomial fit as seen in the figure suggesting that the relationship between the time-averaged temperatures determined using the cold filament approximation and the CDF fits were related independent of position. This relationship was used to determine the transient temperature field as discussed below.

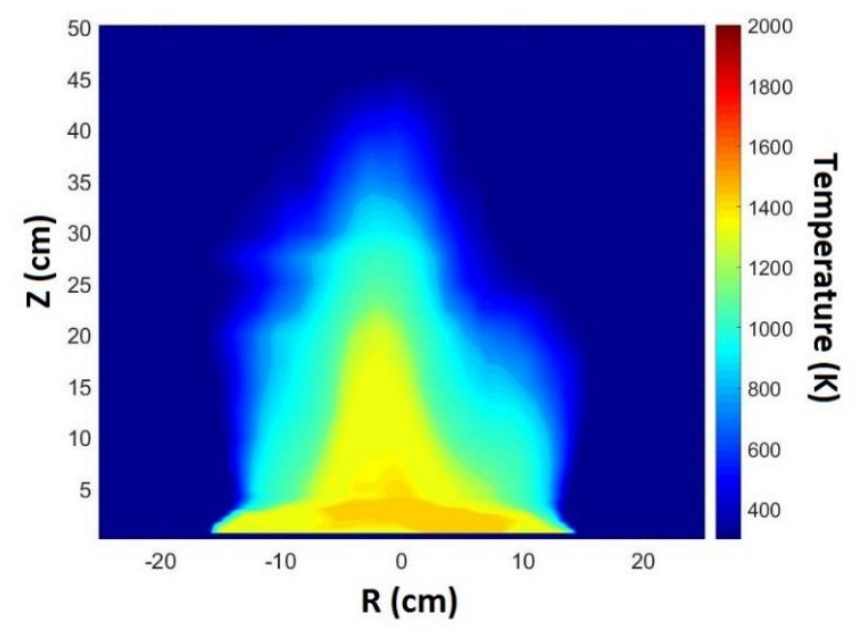

Figure 17. Time-averaged temperature field calculated as a function of position using the CDF fit method.

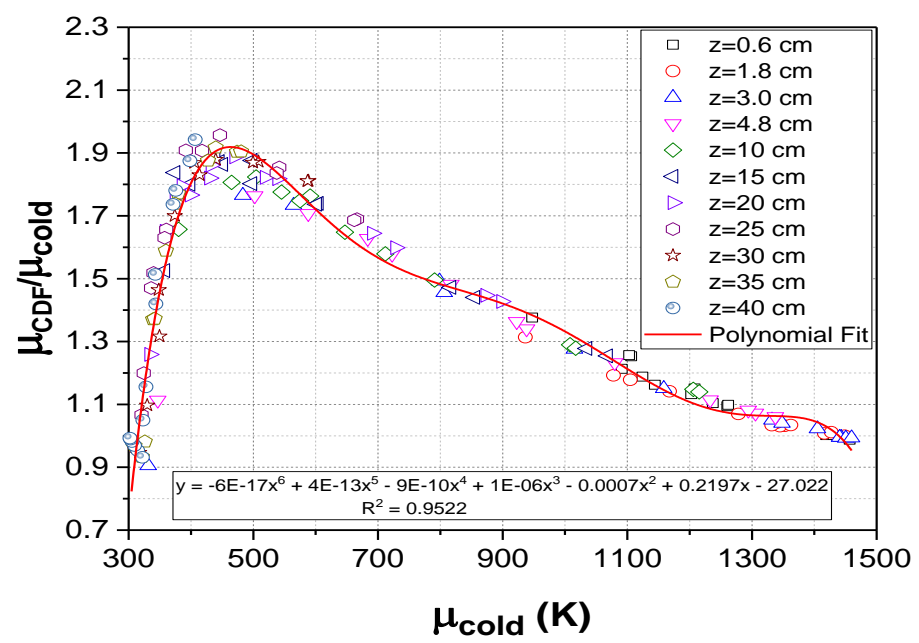

Figure 18. Relationship between the time-averaged temperatures determined from the CDF fit and the cold filament temperature approximation for various radial positions on 11 filaments.

\subsection{Changing Temperature Field}

Using the correlation seen in Figure 18, Figure 19 shows 11 instantaneous false color maps of the temperature field for one puffing cycle of the fire. Here, a reference phase was selected based on the shape of the high temperature core region in the instantaneous flames. The frame order was then defined 
for the subsequent phases in the cycle. The uncertainty in the selection of a reference can be thought of as $\pm 1 / 2$ frame or $0.017 \mathrm{~s}$.

Figure 19 shows false color maps of the instantaneous temperature field for one of the phases (Phase 9) during 10 distinct puffing cycles. There were both differences and similarities in the temperature field. During this phase of the puffing cycle, the fires are tall and sometimes have flames discontinuous from the main body of the fire.
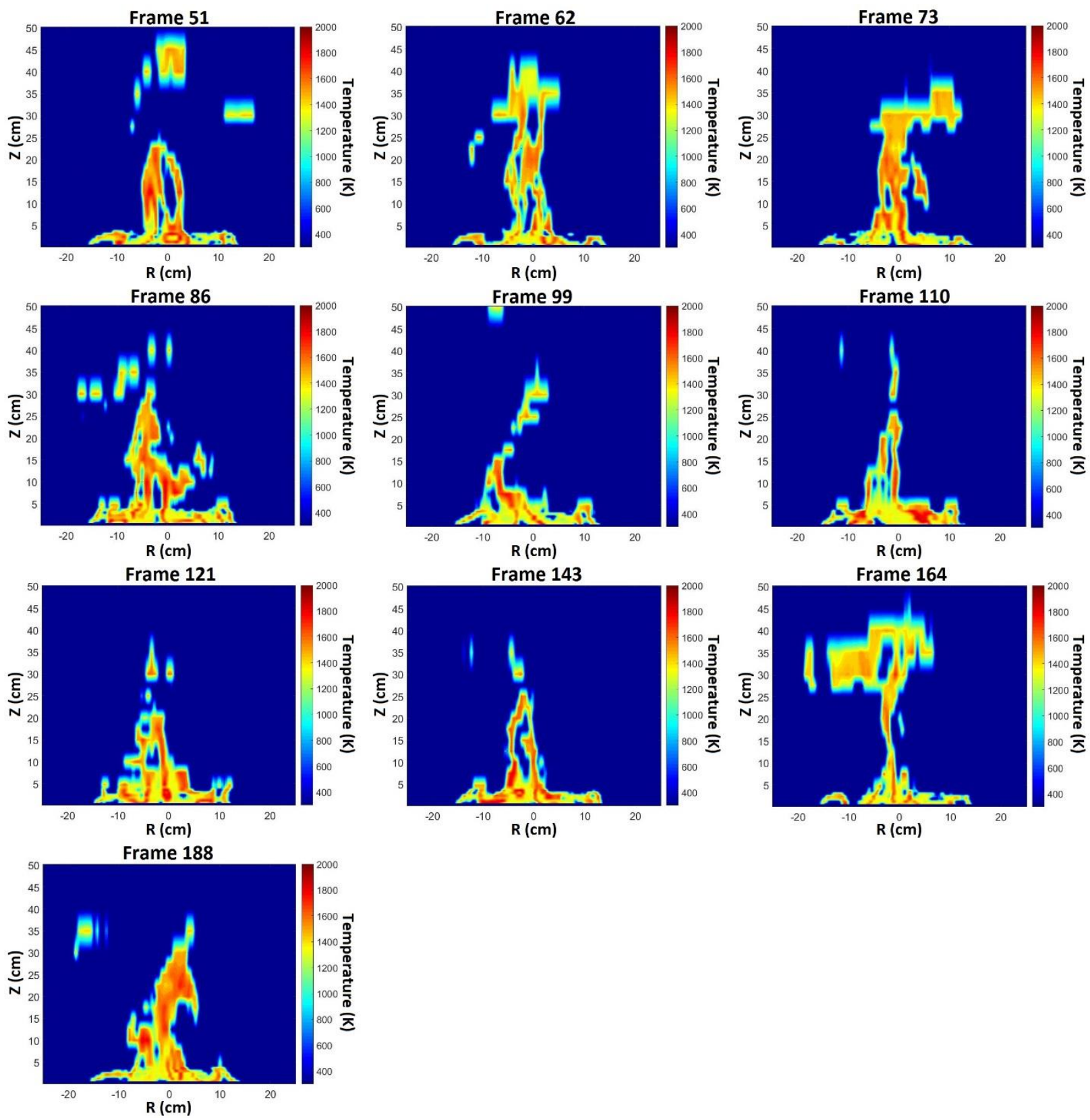

Figure 19. False color maps of the instantaneous temperature field for Phase 9 during 10 distinct puffing cycles.

Figure 20 shows the temperature fields during each of the fire phases averaged over 300 puffing cycles). In the figure, only those puffing cycles that were composed of 11 frames were considered, which was about 
$68 \%$ of the puffing cycles as shown in Figure 12. Information such as this may be of value in model validation studies considering the transient temperature field. As expected, the results in Figure 20 appear smeared when compared to the results in Figure 19. The combined uncertainty, which was larger, was dominated by the uncertainty in the calibration uncertainty and varied as a function of temperature. At $1000 \mathrm{~K}$, the combined uncertainty was approximately equal to $\pm 200 \mathrm{~K}$, whereas at $1500 \mathrm{~K}$, it was equal to $\pm 120 \mathrm{~K}$.
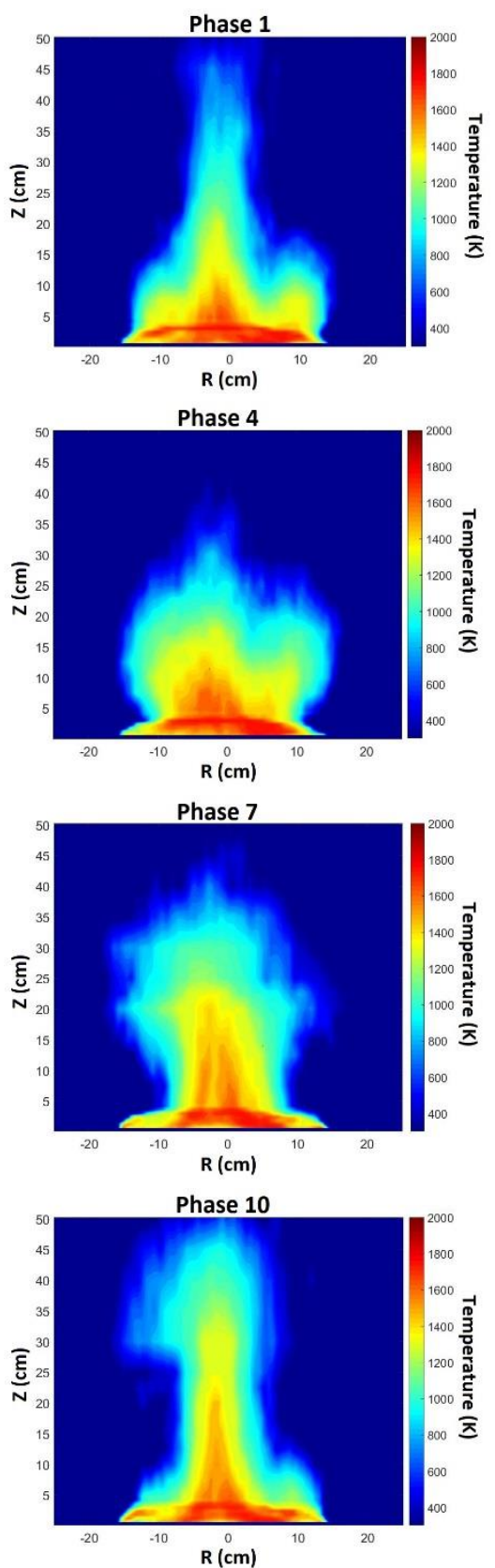
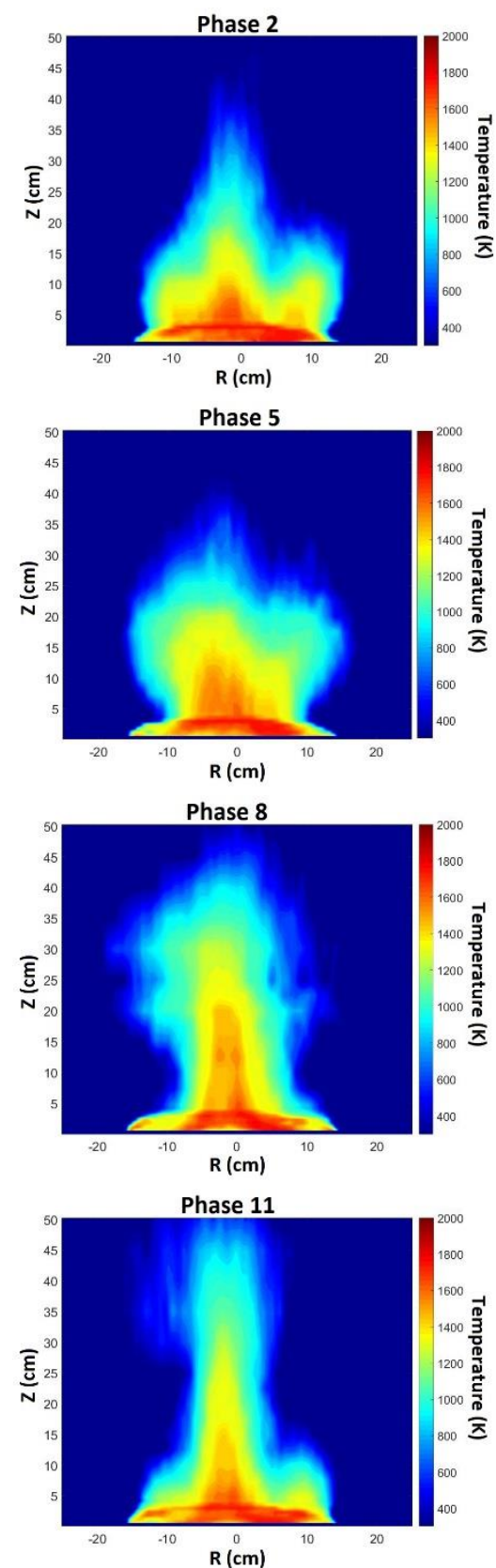
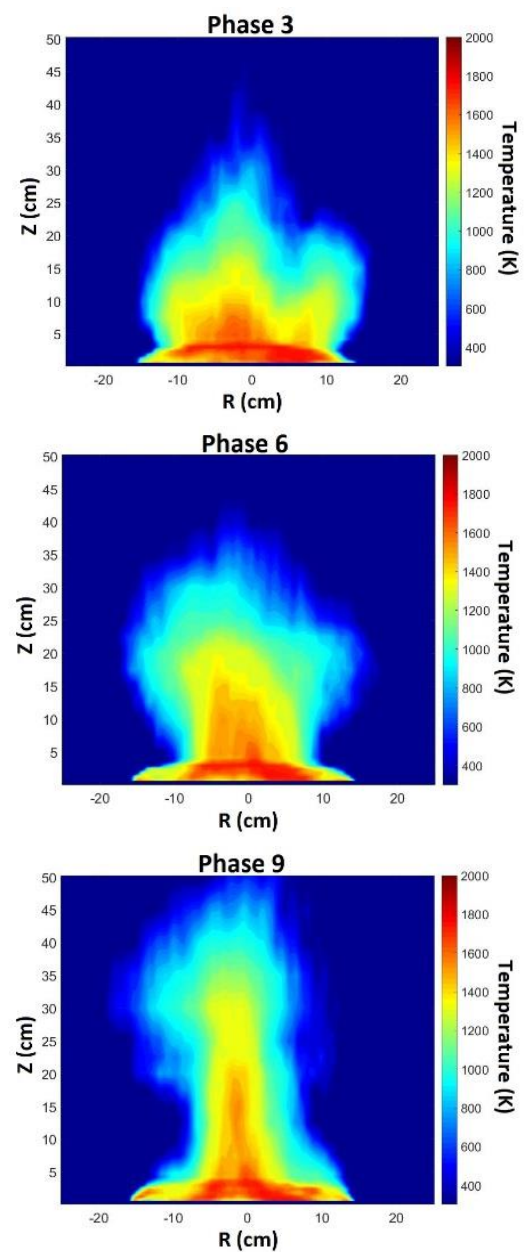

Figure 20. False color map of the transient temperature field for each of 11 phases in the pulsing fire (first row Phases 1 - 3; second row Phases 4 - 6; third row Phases 7 - 9; and forth row Phases 10 - 11). 


\section{SUMMARY AND CONCLUSIONS}

Measurements were made to characterize the time-varying temperature field in a medium-sized methanol pool fire steadily burning in a quiescent environment. Previous pool fire measurements have been made at a single location - temporally uncorrelated with other fire locations. Field measurements enable understanding of the complex thermal structure of a pool fire.

A correlation was developed between the probability density functions of the radiation-corrected thermocouple temperature measurements and the filament luminosity imaged onto an optical camera. The filament was observable by the camera only when its radiative emission was sufficiently large and the gas temperature was sufficiently high, corresponding to a gas temperatures of $1150 \mathrm{~K}$. A percentage of the camera observations exhibited zero grayscale intensity, depending on the instant in time and location in the fire. A calibration was developed that related the gas temperature to the observed filament luminosity. A regression analysis determined the mean temperature and its variance from the cumulative density function of the distribution of calculated filament temperatures for locations in which the probability of grayscale pixel intensity in the camera image was greater than about 0.05 . The resulting fit for time-averaged temperatures followed trends previously reported by Weckman and Strong [9].

A correlation was developed between the fits to the time-averaged gas temperatures and the probability of grayscale pixel intensity in the camera image. This relationship was used to determine the temperature field for eleven phases during the pulsing methanol fire. The results provide insight into the dynamic character of a time-varying pool fire.

The method developed here has several strengths and a few limitations. The method is most easily applied to non-luminous fires burning fuels such as methanol or nitrogen diluted hydrogen, for example, in which the background signal from radiating soot is not a concern. The assumption of a Gaussian fit everywhere in the fire is better in some fire locations than others, which will influence the accuracy of the estimated temperature. The use of an infrared video camera may improve measurement accuracy particularly at lower temperatures.

\section{ACKNOWLEDGEMENTS}

Many thanks are due to Michael Gollner and Peter Sunderland of the University of Maryland at College Park and Howard Baum of NIST for helpful discussions. The authors are grateful to Nippon Carbon Co and Hugh Spilker of COI Ceramics for providing the SiC filament samples. 


\section{REFERENCES}

1. Cetegen, B. M., Ahmed, T., "Experiments on the Periodic Instability of Buoyant Plumes and Pool Fires," Combustion and Flame 93, 157-184 (1993).

2. Peacock, R.D., Reneke, P.A., Forney, G.P., CFAST - Consolidated Model of Fire Growth and Smoke Transport (Version 6), NIST Special Publication 1041r1, March 2013.

http://dx.doi.org/10.6028/NIST.SP.1041r1v

3. McGrattan, K., McDermott, R., Hostikka, S., Floyd, J., Weinschenk, C., Overholt, K., Fire Dynamics Simulator, National Institute of Standards and Technology, Gaithersburg, MD, NIST Special Publication 1019, Sixth Edition, April 2016. http://dx.doi.org/10.6028/NIST.SP.1019

4. Bryant, R.A., Evaluating Practical Measurements of Fire-Induced Vent Flows with Stereoscopic PIV, Proc. Combust. Inst. 33, 2481-2487 (2011). https://doi.org/10.1016/j.proci.2010.06.105

5. Hariharan, S.B., Sluder, E.T., Gollner, M.J., Oran, E.S., Thermal Structure of the Blue Whirl, Proc. Combust. Inst. 37, in press (available online June 2018) https://doi.org/10.1016/j.proci.2018.05.115

6. Hariharan, S.B., The Structure of the Blue Whirl: A Soot-Free Reacting Vortex Phenomenon, Master's Thesis, University of Maryland at College Park, 2017.

7. Pitts, W.M., Thin Filament Pyrometry in Flickering Diffusion Flames, Proc. Twenty-Sixth Sym. (Int.) on Combust, The Combustion Institute, 1171-1179 (1996).

https://ws680.nist.gov/publication/get_pdf.cfm?pub_id=911805

8. Hamins, A., Johnsson, E., Donnelly, M., Energy Balance in a Large Compartment Fire, Fire Safety J. 43, 180-188 (2008).

9. Weckman, E.J., and Strong, A.B., Experimental investigation of the turbulence structure of mediumscale methanol pool fires, Combustion and Flame 105, 245-66 (1996).

10. Hamins, A., Lock, A., The Structure of a Moderate-Scale Methanol Pool Fire, NIST Technical Note 1928, October 2016. https://doi.org/10.6028/NIST.TN.1928

11. Kim, S.C., Lee, K.Y., Hamins, A, Energy Balance in Medium-Scale Methanol, Ethanol and Acetone Pool Fires, submitted to Fire Safety J. (2018).

12. Hamins, A., Klassen, M., Gore, J., Fischer, S., Kashiwagi, T., Heat Feedback to the Fuel Surface in Pool Fires, Combust. Sci. Tech. 97, 37-62 (1993).

13. Klassen, M., Gore, J.P., Structure and Radiation Properties of Pool Fires, NIST Report GCR-94-651, National Institute of Standards and Technology, Gaithersburg, MD, June 1994.

14. Buch, R., Hamins, A., Konishi, K., Mattingly, D., Kashiwagi, T., Radiative Emission Fraction of Pool Fires Burning Silicone Fluids, Combust. Flame 108, 118-126 (1997).

15. Corlett, R.C., Fu, T.M., Some Recent Experiments with Pool Fires, Pyrodynamics 1, 253-269 (1966). 
16. Ichikawa, H., Advances in SiC Fibers for High Temperature Applications, Advances in Science and Technology Online 50, 17-23 (2006). doi:10.4028/www.scientific.net/AST.50.17

17. Goss, L.P., Vilimpoc, V., Sarka, B., Lynn, W.F., J., Thin Filament Pyrometry: A Novel Thermometry Technique for Combusting Glows, ASME J. Eng. Gas Turbines Power 111, 46-52 (1989). http://doi:10.1115/1.3240226

18. Bedat, B., Giovanni, A., Pauzin, S., Thin Filament Infrared Pyrometry: Instantaneous Temperature Profile Measurements in a Weakly Turbulent Hydrocarbon Premixed Flame, Experiments in. Fluids 17, 397-404 (1994). https://doi.org/10.1007/BF01877042

19. OMEGA, Thermocouple Response Time, website consulted 7/1/2018 https://www.omega.com/techref/ThermocoupleResponseTime.html

20. Reotemp Instruments, Type S Thermocouples; website consulted 7/1/2018 http://www.thermocoupleinfo.com/type-s-thermocouple.htm

21. Bergman, T.L., Lavine, A.S., Incropera, F.P., DeWitt, D.P., Fundamentals of Heat and Mass Transfer, $7^{\text {th }}$ ed., Wiley, 2011.

22. Weckman E.J., Sobesiak A., Proc. Twenty-Second Symposium (International) on Combustion, The Combustion Institute, Pittsburgh, 1299 (1988).

23. Hamins, A., Yang, J.C., Kashiwagi, T., An Experimental Investigation of the Pulsation Frequency of Flames, Proc. Twenty-Fourth Sym. (Int.) Combust. 1695-1702 (1992).

24. Petty, M.D., Advanced topics in calculating and using confidence intervals for model validation. Spring Simulation Interoperability Workshop 2013, 194-204 (2013). 